\title{
BMJ Open Patients' perceptions of safety in emergency medical services: an interview study
}

\author{
Anu Venesoja (iD ,1,2 Maaret Castrén, ${ }^{2}$ Susanna Tella, ${ }^{3,4}$ Veronica Lindström ${ }^{5,6}$
}

To cite: Venesoja A, Castrén M, Tella S, et al. Patients' perceptions of safety in emergency medical services: an interview study. BMJ Open 2020;10:e037488. doi:10.1136/ bmjopen-2020-037488

\section{- Prepublication history and} additional materials for this paper is available online. To view these files, please visit the journal online (http://dx.doi org/10.1136/bmjopen-2020037488).

Received 07 February 2020 Revised 21 August 2020 Accepted 17 September 2020

\section{Check for updates}

(c) Author(s) (or their employer(s)) 2020. Re-use permitted under CC BY-NC. No commercial re-use. See rights and permissions. Published by BMJ.

${ }^{1}$ South Karelia Social and Healthcare District,

Lappeenranta, Finland

${ }^{2}$ Department of Emergency

Medicine and Services, Helsinki

University Hospital, Helsinki

University, Helsinki, Finland

${ }^{3}$ Faculty of Social Services and Health Care, LAB University of

Applied Sciences, Lappeenranta, Finland

${ }^{4}$ Department of Nursing Science, Faculty of Health Sciences, University of Eastern Finland, Kuopio, Finland

${ }^{5}$ Samariten Ambulance, Stockholm, Sweden

${ }^{6}$ Department of Neurobiology, Care Sciences, and Society Division of Nursing Stockholm, Karolinska Institutet, Solna, Sweden

Correspondence to

Anu Venesoja;

anu.venesoja@helsinki.fi

\section{ABSTRACT}

Background Research on patient safety in emergency medical services (EMS) has mainly focused on the organisation's and/or the EMS personnel's perspective. Little is known about how patients perceive safety in EMS. This study aims to describe the patients' experiences of their sense of safety in EMS.

Methods A qualitative design with individual interviews of EMS patients $(n=21)$ and an inductive qualitative content analysis were used.

Results Patients' experiences of EMS personnel's ability or inability to show or use their medical, technical and driving skills affected the patients' sense of safety. When they perceived a lack of professionalism and knowledge among EMS personnel, they felt unsafe. Patients highlighted equality in the encounter, the quality of the information given by EMS personnel and the opportunity to participate in their care as important factors creating a sense of safety during the EMS encounter. Altogether, patients' perceptions of safety in EMS were connected to their confidence in the EMS personnel.

Conclusions Overall, patients felt safe during their EMS encounter, but the EMS personnel's professional competence alone is not enough for them to feel safe. Lack of communication or professionalism may compromise their sense of safety. Further work is needed to explore how patients' perceptions of safety can be used in improving safety in EMS.

\section{BACKGROUND}

'To err is human', ' but it can at worst cause disastrous results for patients seeking care and for the organisation caring for them. Therefore, systematic development and research are needed to ensure and improve patient safety and quality of care. Errors are described as being usually caused by faulty systems, processes or conditions in the organisation rather than by individual healthcare workers, and thus, all healthcare actors, including patients, should be involved in developing the safety culture in healthcare. Global recommendations and guidelines to improve patient safety include the patients as active team members whenever possible. ${ }^{1-3}$ Patients' experiences of difficulties and

\section{Strengths and limitations of this study}

This study provides knowledge about what patients consider important for feeling safe in the emergency medical services (EMS).

- Detailed, rich information was captured and analysed of individual patient perceptions of their safety during their interactions with EMS.

- A limitation is that the interviews were done in a small hospital district, which could limit the transferability of the results.

harms can provide information about safety, which is not obvious to healthcare staff. ${ }^{4}$

The WHO has defined the term safety culture as the product of individual and group values, attitudes, perceptions, competencies and patterns of behaviour that determine the commitment to, and the style and proficiency of, an organisation's health and safety management. According to WHO, another definition for safety culture is an integrated pattern of individual and organisational behaviour, based on shared beliefs and values, that continuously seeks to minimise patient harm, which may result from the processes of care delivery. ${ }^{5}$ When researchers use the term 'patient safety culture', they define sections of safety culture that have an impact on patient safety. ${ }^{6-9}$ Considering this relationship between safety culture and patient safety culture, it is essential to recognise how relationship affects the patients' perceptions of safety. In this study, safety in emergency medical services (EMS) is explored from the patients' perspective. The EMS includes healthcare professionals who respond to emergency calls, assessing, treating and transporting patients to healthcare providers such as the emergency department (ED).

\section{Safety and patient safety in EMS}

By nature, EMS can be considered a challenging and constantly changing environment compared with other emergency care 
settings such as hospitals. The hospital environment is especially built for patient care, whereas the EMS personnel treat the patients in their homes, in public, inside the ambulance or outdoors. Because the environment is not always predictable in EMS, it could compromise the safety of both EMS personnel and patients. Transporting a patient to hospital by ambulance could also be a hazardous situation. The risks of traffic accidents are known to increase if driving with lights and sirens. ${ }^{10} 11$ There is some evidence that safety culture and patient and EMS provider safety outcomes are interrelated. EMS personnel who reported an error or adverse event (AE) evaluate safety culture lower than those who did not report an error or AE. Furthermore, EMS personnel who reported safety-compromising behaviour evaluate safety culture lower than those who did not. ${ }^{12}$

Otherwise, patient safety studies within the EMS setting have mainly investigated AE, mishaps, near-misses, occupational hazards and patient safety or quality of care, and these previous studies have mainly focused on the organisation's or EMS personnel's perspective and ignored the patients' point of view on safety. ${ }^{13-17}$ Patient safety from the their own viewpoint has mainly been investigated in hospital settings, showing that they give valuable insights into improving or assessing patient safety. ${ }^{18-20}$ As the EMS personnel sometimes has to work in a challenging environment, including risks of driving hazards, there is a need to investigate the patients' perceptions of safety in the EMS. Therefore, the aim of this study was to describe the patients' perceptions of safety in the EMS.

\section{METHODS}

A qualitative study design with individual interviews was used to explore patients' perceptions of safety in the EMS.

\section{Setting}

This study was carried out in Finland, where the hospital districts are responsible for organising the EMS. The Finnish EMS consists of advanced-level ambulances and basic-level ambulances, and every hospital district must have at least one EMS officer (operational supervisor of the shift, participates in challenging tasks). The advancedlevel ambulances are staffed with two prehospital nurses or one prehospital nurse and another qualified person, for example, nurse or other healthcare professional or rescue worker. The education level among advancedlevel prehospital nurses is at least a registered nurse (3.5 years) with advanced life support education (1 year alongside the work) or a prehospital nurse (4 years). Basiclevel ambulances are manned by at least one emergency medical technician (EMT). ${ }^{21} 22$ The EMS officer should be an advanced-level prehospital nurse with operative leadership education (eg, masters' degree or 1 year operational leadership education) and leadership experience. The highest educated EMS personnel is always responsible for patient care, but when the patient is assessed as low priority, a nurse, EMT or other healthcare professional can attend to the them during transport.

The healthcare district this study was conducted in is eastern Finland and it covers approximately 132000 inhabitants. There is one central hospital in the district and ambulance services cover an area $6872.10 \mathrm{~km}^{2}$, including both rural and urban regions. Ambulance transports vary between 1 and over $100 \mathrm{~km}$. In 2017, there were about 22100 EMS requests in the area according to official statistics. At present (in 2018), there are one EMS officer, 11 ambulances, all of them advanced level ambulances, and in addition there are two units with the assignments to treat and evaluate low-priority patients at home. The units have the same equipment as the ambulance and point-of-care devices, but they are not capable of transporting the patient.

\section{Data collection and participants}

Data collection was undertaken at the central hospital ED, where patients are transported by EMS. Data were collected via semistructured interviews during a 2-week period in March 2018. The interviews were conducted by the first author, a prehospital nurse with 20 years' working experience in the EMS, who has not had any professional or personal contact with the participants beforehand. Purposeful sampling ${ }^{23}$ was used, aiming to achieve variation (gender, age, urban/rural area, primary condition) among participants without risking patient safety. The inclusion criteria were as follows: the patient was transported by the EMS to the ED after an emergency call to the emergency response centre (ERC). The patient was assessed as low priority in the ED or the patient was transported to the hospital as high priority, but the priority was assessed as low after treatment in the ED. The patients needing urgent treatment in the ED, patients under the influence of alcohol (based on ED nurses' assessment) or drugs and interhospital transports were excluded. Additional exclusion criteria were being younger than 18 years of age, incapability of communicating in Finnish or presence of dementia, confusion or terminal disease. ED nurses identified eligible participants. The first author received a list of eligible participants from the ED nurse, gave oral and written information about the study and asked about participation after patients had received their initial assessment and treatment at the ED.

All interviews were performed on weekdays between 8:00 to 16:00, although some of the interviewed patients had been transported to the ED in the night time. The first, second and last authors (the first and last authors with working experience in EMS as prehospital nurses, and the second author with experience as an EMS physician) together devised the interview questions. The interviews started with an open-ended question: 'Can you tell me about your experience of the EMS encounter?' To encourage patients to share their experiences, additional questions were asked concerning waiting time, assessment, treatment, transportation and the handover at the ED. The interviews were concluded by asking the 
patients to describe what made them feel safe or insecure during the EMS encounter. The interview guide is presented in online supplemental file 1 . The authors held multiple discussions during the data collection. The interviews lasted between 10 and $20 \mathrm{~min}$. The interviews continued until no new information was obtained during the interviews. The variations in the interviews started to be limited during interview 15 , but six more interviews were conducted aiming to ensure that no new variations would emerge. All the interviews were recorded with a digital recorder and transcribed verbatim by the first author. All the transcriptions were anonymised. Two of the interviews were translated from Finnish into English to achieve transparency among all authors participating in the study.

\section{Patient and public involvement}

The patients or the public were not involved in the design and conduct of this study.

\section{Data analysis}

An inductive qualitative content analysis was used to analyse the data. ${ }^{24}$ The analysis began after all interviews had been listened to and transcribed. The text was then read several times to obtain a sense of the whole and to identify the patients' expressions about their perceptions of safety in the EMS. The expressions were single words or short sentences. The third author, who had no experience in EMS, but had working knowledge of patient safety research, read the transcripts with the aim of increasing the reliability of the process and verifying the first phase of open coding, in which similar expressions received the same open code. The coding was made without using any software for analysis. An example of the coding tree is presented in online supplemental file 2.

After the open coding, the codes were collected into a sheet with other related codes. These coding sheets were then abstracted into subcategories, which were grouped into generic categories and finally into the main category. During the analysis, there was a recurrent movement between the whole and the parts. The authors held multiple discussions to ensure the reliability and credibility of the analysis, keeping the balance between their preunderstanding and openness to the content during the analysis. In every phase, the analysis continued until
Table 1 Description of patients

Female $(n=12)$ Male $(n=9)$

\begin{tabular}{lll}
\hline Age range (mean) & $44-91$ (74.5) & $41-86$ (68.1) \\
Transported from urban area & 7 & 5 \\
Transported from rural area & 5 & 4
\end{tabular}

Primary condition as patients described

\begin{tabular}{lll} 
Breathing difficulties & 4 & 1 \\
\hline Cardiac-related symptoms & 3 & 2 \\
\hline Gastrointestinal problems & 2 & 1 \\
\hline Lower body pain & 1 & 1 \\
Minor injury & - & 2 \\
Neurological symptoms & - & 2 \\
Missing data & 2 & - \\
\hline
\end{tabular}

consensus between the researchers was reached. The last phase in the analysis was the conceptualisation of the results, displayed in figure 1.

\section{RESULTS}

In total, 22 patients were asked to participate, 21 of whom agreed to participate in the study. One male refused the interview without providing a reason. Some of the patients had used EMS more than once and for some of them, this was a first contact to the EMS. The main reason for seeking EMS care was cardiac-related symptoms or breathing difficulties, as displayed in table 1. Two of the patients did not describe their health problem or the reason for requesting an ambulance.

The main category Patients' confidence in the EMS shows that the patients feel safe in the EMS and have confidence in EMS personnel. The patients' confidence in the EMS personnel were divided in two generic categories: EMS personnel's social skills and circumstantial factors affecting patients' care. EMS personnel's social skills and professional competence consist of subcategories equal treatment, information, involvement in care decisions and EMS personnel's professional competence. Circumstantial factors affecting patients' care is composed of subcategories environmental factors and EMS personnel's driving skills. (figure 1). The generic categories with

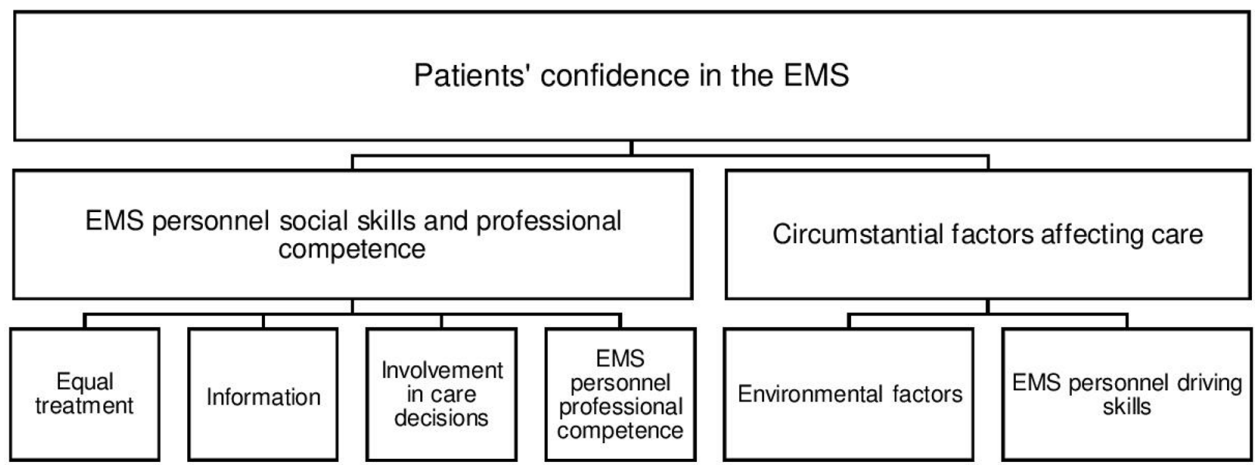

Figure 1 Overview of the categories. EMS, emergency medical services. 
their subcategories are presented below with illustrative quotations.

\section{EMS personnel's social skills and professional competence}

The EMS personnel's social skills and professional competence that affected patients' sense of safety in EMS included being treated equally, receiving information, being involved in their care and getting professional treatment.

\section{Equal treatment}

According to the patients, equal treatment and a reliable patient-EMS personnel relationship generated a sense of safety in the EMS. The patients noted that it is essential that the EMS personnel's behaviour is calm, natural and friendly. They expressed that a bit of humour and small talk during the care lighten the atmosphere and help to create a good patient-EMS personnel relationship.

They didn't feel like officials. They were like human to human. (Pt5)

On the other hand, patients said that they felt insecure or that the EMS personnel acted in a condescending way when the personnel's behaviour was rushed, negative or too official, or when the personnel lacked communication skills. The patients also stated that the EMS personnel did not always take their concerns seriously and sometimes ignored them altogether. This was reflected in how patients described situations where their mental and/ or physical condition created a feeling of insecurity, for example, if they had difficulties with breathing, felt lonely or had to wait for the ambulance for a long time. Feeling insecure because of condescending treatment caused a sense of being unsafe among the patients.

Waiting is the worst, especially if you are alone and there isn't anyone with you. (Pt6)

\section{Information}

Most patients mentioned that the EMS personnel handed over enough information about the assessments, a student's presence, environmental conditions, treatment and medication as well as about driving with lights and sirens on. In addition, if the EMS personnel had contacted the hospital beforehand, the patients expressed that the information had transferred to the hospital personnel. The patients described that in these situations, their treatment in the hospital started smoothly and quickly. However, some patients mentioned not getting enough information. Usually, the lack of information concerned what the EMS personnel has assessed, the assessments results or the patient's medication during care. Even these patients maintained confidence in the EMS personnel and their professionalism because of the feeling that they received help from EMS personnel. Lack of information thus had negligible impact on patients' feelings of safety in the EMS.
Ambulance personnel interviewed me and they took all sorts of assessments and I don't know all the assessments they took. (Pt13)

Involvement in care decisions

According to the patients, their involvement in care decisions varied. The patients' possibility to affect their transport position had an impact on their sense of safety. Especially the ones suffering from breathing problems stated that they wanted to sit on the seat rather than lay on the stretcher even if they were placed in an upright position. However, EMS personnel usually ignored this wish without explaining why it was not possible. Although some of the patients said that they did not have the chance to influence how they were moved to the ambulance or what position or where to stay during the transport, they did not automatically consider it negative.

They didn't let me walk anymore, they were pushing (with the stretchers) the old granny... it sort of gives a nice feeling that somebody is still taking care of the old granny. (Pt5)

In some situations regarding safety, the patients took an active role. For example, they asked the EMS personnel to put safety belts on or they asked to reduce ambulance speed if they felt that the speed compromised their safety.

I said that at least put the seatbelt on me. If you drive off the road, I fly out of here (from the stretchers) because I don't have the seatbelt on. (Pt10)

\section{EMS personnel's professional competence}

Patients stated that EMS personnel's professional competence made them feel safe during care. According to them, good professional competence means asking questions related to their health problems, background information about previous illnesses, medication, home situation, and so on, and taking assessments and giving medication when needed. These factors made them feel that the treatment had started immediately and that the EMS personnel were interested in their health problem. Furthermore, the patients mentioned that when the EMS personnel supervised and gave guidance to the student it also had an effect on the patient's perception of the EMS personnel's professional competence. They noted that the EMS personnel mainly had good professional competence from their point of view.

The guys inserted an IV (intravenous cannula) and did assessments. Very professional personnel inserted the IV into my forearm, so they are very well educated. (Pt11)

They took care of me and measured my blood pressure and gave me the medication orally and that made me feel safe. (Pt8)

However, some of the patients perceived that the EMS personnel lacked professional competence, and this 
affected their sense of safety. This situation occurred when the EMS personnel were uncertain of what had caused the patients' health problem or when the patient became aware that the EMS personnel had a lack of knowledge, for example, when the only solution to the problem in the EMS personnel's view was to transport the patient to the hospital. In addition, when the personnel were unable to put in an intravenous cannula, the patients interpreted it as a lack of professional competence. These factors made the patient feel uncertain and unsafe.

They said that they don't understand, and they brought me here (hospital)... they tried to insert an IV in my forearm and it failed. (Pt3)

\section{Circumstantial factors affecting care}

Environmental factors (eg, road and weather conditions, ambulance suspension and conditions inside the ambulance during the transport) and driving skills create the circumstances where the EMS patients get treatment. These circumstantial factors were highlighted when the patients talked about their perceptions of factors affecting the care and sense of safety in EMS.

\section{Environmental factors}

Environmental factors markedly affect patients' feelings of safety in EMS. They feel that EMS provides an essential public safety function. Almost all of the patients interviewed had some preconceived notions of how the EMS works, expectations based on their own perceptions or on how the service has been described in the media. Quick response times increase their perceptions of safety. However, the experience of a quick response time varied between the patients. They mentioned that they felt safe while the ambulance transported them to hospital. They also described a feeling of relief and security when the EMS personnel arrived and brought help to them with good equipment.

Because I know that every time when I call an ambulance, help is near. (Pt13)

Some environmental issues reduced the patients' feeling of safety or made them uncomfortable. Uncomfortable and narrow stretchers and difficulties in getting inside the ambulance impair the experience of the care. The experience of feeling bad increased if the temperature was too hot or too cold during the transport. Bad, bumpy roads or poor suspension in the ambulance also made patients feel worse.

Why did the ambulance have such bad and noisy suspension? Was the road so bad or was it the ambulance suspension? (Pt10)

\section{EMS personnel's driving skills}

For the most part, the patients felt that the EMS personnel had good driving skills, reflected in 'smooth and fast transportation' or not driving too fast. Furthermore, if the driver took notice of the weather and road conditions and adjusted the driving style accordingly, the patient had an impression of good driving skills and safe transportation. However, some of the patients felt unsafe and insecure if the ambulance's speed was too high, especially if the weather conditions were bad or the roads were slippery or uneven.

It was hailing, they were the size of ping pong balls, and other cars had stopped at the roadside but the ambulance was going very fast. (Pt10)

\section{DISCUSSION}

Overall, the interviewed patients appeared to feel safe in the EMS and to have confidence in EMS personnel. Clearly, confidence in the care provider is the main factor affecting patients' sense of safety in the EMS. In addition, medical knowledge and driving skills are directly related to a positive sense of safety. However, the EMS personnel's professional competence and good driving skills are meaningless in maintaining the patients' confidence if the EMS personnel does not treat them in an equal and humane manner. Therefore, EMS personnel should become more aware of their social interactions and their importance to patients' perception of safety. In healthcare overall and in the EMS setting, it is crucial that healthcare workers support patient involvement in care decisions and provide relevant information to the patients. By seeing the patient as a team member and involving them in their care, ${ }^{19}{ }^{25}$ the EMS personnel can create a psychologically safe environment for the patients. Patients then are more likely to talk about their concerns, to get an experience of interaction and to feel safe in the EMS encounter. In previous research, the Finnish patient safety experts stated that trust in the healthcare professionals and their attitudes towards patient participation in general are important, when involving patients in improving patient safety. ${ }^{26}$

In this study, the perception of equality, the possibility to get information and the involvement in care decisions affected the patient's sense of safety in the EMS. A previous study ${ }^{27}$ showed that shared information and being treated in a friendly and respectful manner are important according to patients. If they feel objectified by the EMS personnel, this may cause a feeling of 'suffering from care', ${ }^{28}$ leading to a sense of unsafety. Previous knowledge of patient experiences of safety in hospital settings ${ }^{18-20}$ highlights that being treated equally is important to patients, and based on our findings this is also true in the context of EMS.

In other healthcare settings, researchers establish positive associations between the patient experiences and patient safety and clinical effectiveness. ${ }^{29}$ From the experiences, valuable information emerged on how to improve patient safety and the patient encounter in EMS. The EMS personnel's clinical judgement was important when patients described what makes them feel safe when 
cared for by EMS personnel. On the other hand, some of the patients had experienced, especially with driving, a situation that could have compromised the safety of the patient and the EMS personnel. A previous study reveals that EMS users value a short waiting time, confidence, professionalism and communication. ${ }^{30}$ Our study points out that these same factors also influence their perceptions of their safety. However, in our study a short waiting time according to patients ranged from a few to $30 \mathrm{~min}$.

In some respects, our findings are in line with the results described in former patient safety culture studies. ${ }^{6-9}$ The categories equal treatment, information and involvement in care decisions reflect both the 'social process' and the 'psychological dimension ${ }^{, 7}$ or teamwork, communication and patient-centredness described in other studies. ${ }^{689} \mathrm{On}$ the other hand, the categories EMS personnel's professional competence, environmental factors and EMS personnel's driving skills reflect the 'organisational dimension ${ }^{7}$ or leadership and evidence-based healthcare described in the other studies. ${ }^{68}{ }^{6}$ Like a study conducted in hospital setting suggests, ${ }^{31}$ error management should promote developing a strong safety culture that affords the patient a role in promoting safety in their care. Our study highlights the gap between what safety means to the EMS personnel or the EMS organisation and what kind of perceptions patients had safety in the EMS encounter. Patient perception of safety in the EMS is not the same as actually receiving safe care. Therefore, EMS organisations and EMS personnel must continue to develop the other safety elements in the EMS.

Furthermore, based on this study and a former study, ${ }^{32}$ EMS personnel, EMS organisations and vocational training providers need additional knowledge about factors affecting patients' sense of safety in the EMS. The EMS personnel require more education to improve their social skills and to be able to foster psychological safety for the patient. The curriculum in EMS personnel training should thus be expanded to include development of social skills. Therefore, in the future, it could be beneficial to explore the social factor between EMS personnel and the patients by using ethnographic framework within observational study.

\section{Study strengths and limitations}

It could be a strength or a limitation that the researchers had a deep preunderstanding of the research topic. Our deep theoretical and clinical experience helps us to understand patients' experiences of the EMS and also to put these into a clinical context despite the short interviews. However, theoretical and clinical experience could also cause a bias via a lack of openness to the subject. To reduce this potential bias, we moved back and forth between the interviews and the expressions and between the categories and the interviews during the analysis. In addition, one of the researchers had no experience with EMS, but had working knowledge of patient safety, and this reduced the risk of bias caused by preconceptions.
The patients were recruited from only one healthcare district area, which could reduce the transferability of the results. However, patients' characteristics cover common EMS patient groups according to the ERC official statistics, and therefore, it is reasonable to think that the results can be transferred to a similar context. According to the exclusion criteria, we did not interview highpriority patients suffering for multiple traumas or other life-threatening conditions or interhospital transfers. These patients could have given valuable information on their perceptions of safety when EMS personnel must use, for example, support equipment and different kinds of transfer methods.

The interviews were performed when the patient was admitted to the ED. This may also be considered a limitation or a strength: a limitation due to the patient's experiences of illness, a strength due to their memory of the EMS personnel and the EMS encounter being fresh and unaffected by other people's opinions. Because of the timing of the interviews, one might assume that the care in the EMS was still in the patients' recent memory. The short duration of the interviews may be a limitation and may have been caused by the patients' illness or fatigue. It is possible that the short duration would limit the depth of understanding.

Even though the interviews were done alone with the patient, it is possible that the patients were hesitant to openly share their views. There could have been barriers to the patients disclosing their concerns caused by, for instance, 'I do not want to be a troublemaker', 'I do not know how to raise my concern' or 'I do not want to harm my relationship with members of the medical team'.33 To reduce these concerns, the interviewer introduced herself as a researcher, wore casual attire and informed the patient that interviews are analysed anonymously. Moreover, we informed the patients that participating or withdrawing or anything that they say will not influence their treatment in the hospital or EMS. Despite certain limitations, this study offers valuable insights into patients' perceptions of safety in EMS.

\section{CONCLUSIONS}

The EMS personnel's social interactions seem to be associated with patients' perceptions of safety. Thus, more attention should be directed to their social skills and their ability to create a psychologically safe environment for the patient. In addition, this study adds to the knowledge about the factors contributing to or reducing patients' perception of safety when attended to by EMS personnel. This information is valuable when EMS organisations design methods to involve patients in developing their safety performance.

\section{Twitter Susanna Tella @susanna_tella}

Acknowledgements We thank the EMS patients who shared their experiences with us. We also thank the emergency department nurses for valuable help in recruiting patients. Thanks to the Spoken company for the copyediting services. 
Contributors All authors contributed to this study as follows: study design (AV, VL, $\mathrm{MC})$, data collection (AV), data analysis (AV, VL, ST) and writing the manuscript (AV, VL, MC, ST). All authors read and approved the final manuscript.

Funding Funding was provided by Finnish State Research Funding.

Competing interests None declared.

Patient consent for publication Not required.

Ethics approval This study was approved by the Ethics Committee of Helsinki University Hospital (HUS/3529/2017). The patients received written information about the purpose of the study with contact information for the responsible researcher, and they had the possibility to ask the first author questions about the research. The patients filled out a form affirming their voluntary participation in the study. The patients were informed that they have the right to withdraw from the study at any phase. During the interviews, the first author observed the patients and discontinued the discussion if any changes occurred in the patient's physical or mental condition.

Provenance and peer review Not commissioned; externally peer reviewed.

Data availability statement The data used and analysed during the study are available from the corresponding author on reasonable request.

Supplemental material This content has been supplied by the author(s). It has not been vetted by BMJ Publishing Group Limited (BMJ) and may not have been peer-reviewed. Any opinions or recommendations discussed are solely those of the author(s) and are not endorsed by BMJ. BMJ disclaims all liability and responsibility arising from any reliance placed on the content. Where the content includes any translated material, BMJ does not warrant the accuracy and reliability of the translations (including but not limited to local regulations, clinical guidelines, terminology, drug names and drug dosages), and is not responsible for any error and/or omissions arising from translation and adaptation or otherwise.

Open access This is an open access article distributed in accordance with the Creative Commons Attribution Non Commercial (CC BY-NC 4.0) license, which permits others to distribute, remix, adapt, build upon this work non-commercially, and license their derivative works on different terms, provided the original work is properly cited, appropriate credit is given, any changes made indicated, and the use is non-commercial. See: http://creativecommons.org/licenses/by-nc/4.0/.

ORCID iD

Anu Venesoja http://orcid.org/0000-0003-4634-1006

\section{REFERENCES}

1 Institute of Medicine (US) Committee on Quality of Health Care in America.Kohn LT, Corrigan JM, Donaldson MS, eds. To err is human: building a safer health system. Washington DC, US: National academies press, 2000.

2 World Health Organization. Exploring patient participation in reducing health-care related safety risks. World Health organization, Copenhagen, 2013. Available: http://www.euro.who.int/_data/ assets/pdf file/0010/185779/e96814.pdf

3 World Health Organization. Patient engagement. Technical series on safer primary care. Geneva: WHO, 2016. https://apps.who.int/iris/ bitstream/handle/10665/252269/9789241511629-eng.pdf

4 Vincent C, Burnett S, Carthey J. Safety measurement and monitoring in healthcare: a framework to guide clinical teams and healthcare organisations in maintaining safety. BMJ Qual Saf 2014;23:670-7.

5 World Health Organization (WHO). Conceptual framework for the International classification for patient safety, version 1.1, final technical report, 2009. Available: https://apps.who.int/iris/bitstream/ handle/10665/70882/WHO_IER_PSP_2010.2_eng.pdf?sequence=1

6 Sammer CE, Lykens K, Singh KP, et al. What is patient safety culture? A review of the literature. J Nurs Scholarsh 2010;42:156-65.

7 Reiman T, Pietikäinen E, Oedewald P. Multilayered approach to patient safety culture. Qual Saf Health Care 2010;19:e20.

8 Stavrianopoulos T. The development of patient safety culture. Health Sci J 2012;6:201.

9 Reis CT, Paiva SG, Sousa P. The patient safety culture: a systematic review by characteristics of hospital survey on patient safety culture dimensions. Int J Qual Health Care 2018;30:660-77.
10 Holgate R. The opinion of emergency medical service personnel regarding safety in pre-hospital emergency care practice. Johannesburg: University of the Witwatersrand, 2015. https:// pdfs.semanticscholar.org/fbb6/a55bfbf33da065422982f16a79de 87fd8a46.pdf

11 Brice JH, Studnek JR, Bigham BL, et al. Ems provider and patient safety during response and transport: proceedings of an ambulance safety conference. Prehosp Emerg Care 2012;16:3-19.

12 Weaver MD, Wang HE, Fairbanks RJ, et al. The association between EMS workplace safety culture and safety outcomes. Prehosp Emerg Care 2012;16:43-52.

13 Salminen-Tuomaala M, Leikkola P, Paavilainen E. Patient and staff safety incidents and near misses in out-of-hospital emergency care. Emergency Med 2014;4:219.

14 Salminen-tuomaala M, Leikkola P, Paavilainen E. Emergency health care professionals' experiences of factors that influence care quality and safety. Clin Nurs Stud 2015;3:60-8.

15 Bigham BL, Buick JE, Brooks SC, et al. Patient safety in emergency medical services: a systematic review of the literature. Prehosp Emerg Care 2012;16:20-35.

16 Coster JE, Irving AD, Turner JK, et al. Prioritizing novel and existing ambulance performance measures through expert and lay consensus: a three-stage multimethod consensus study. Health Expect 2018;21:249-60.

17 Fairbanks RJ, Crittenden CN, O'Gara KG, et al. Emergency medical services provider perceptions of the nature of adverse events and near-misses in out-of-hospital care: an ethnographic view. Acad Emerg Med 2008;15:633-40.

18 Rainey $\mathrm{H}$, Ehrich K, Mackintosh $\mathrm{N}$, et al. The role of patients and their relatives in 'speaking up' about their own safety-a qualitative study of acute illness'. Health Expect 2015;18:392-405.

19 Sahlström M, Partanen P, Azimirad M, et al. Patient participation in patient safety-an exploration of promoting factors. J Nurs Manag 2019;27:84-92

20 McTier L, Botti M, Duke M. Patient participation in medication safety during an acute care admission. Health Expect 2015;18:1744-56.

21 Health Care Act 1326:2010. Ministry of social affairs and health, Finland 2013.

22 Ministry of Social Affairs and Health decree of prehospital emergency care 585. Ministry of social affairs and health, Finland 2017.

23 Patton MQ. Qualitative research and evaluation methods: integrating theory and practice. 4th edn. California, USA: SAGE Publications, Inc, 2015: 52-3.

24 Elo S, Kyngäs $\mathrm{H}$. The qualitative content analysis process. J Adv Nurs 2008:62:107-15.

25 Edmondson A. Psychological safety, trust and learning: a group-leve lens. In: Kramer R, Cook K, eds. Trust and distrust in organizations: dilemmas and approaches. eds. New York: Russell Sage Foundation, 2004: 239-72.

26 Sahlström M, Partanen P, Rathert C, et al. Patient participation in patient safety still missing: patient safety experts' views. Int J Nurs Pract 2016;22:461-9.

27 Bishop AC, Macdonald M. Patient involvement in patient safety: a qualitative study of nursing staff and patient perceptions. J Patient Saf 2017;13:82-7.

28 Ahlenius M, Lindström V, Vicente V. Patients' experience of being badly treated in the ambulance service: a qualitative study of deviation reports in Sweden. Int Emerg Nurs 2017;30:25-30.

29 Doyle C, Lennox L, Bell D. A systematic review of evidence on the links between patient experience and clinical safety and effectiveness. BMJ Open 2013;3:e001570.

30 Togher FJ, O'Cathain A, Phung V-H, et al. Reassurance as a key outcome valued by emergency ambulance service users: a qualitative interview study. Health Expect 2015;18:2951-61.

31 Sahlström M, Partanen P, Turunen H. Safety as experienced by patients themselves: a Finnish survey of the most recent period of care. Res Nurs Health 2014;37:194-203.

32 Sjölin $\mathrm{H}$, Lindström V, Hult $\mathrm{H}$, et al. What an ambulance nurse needs to know: a content analysis of curricula in the specialist nursing programme in prehospital emergency care. Int Emerg Nurs 2015;23:127-32.

33 Bell SK, Roche SD, Mueller A, et al. Speaking up about care concerns in the ICU: patient and family experiences, attitudes and perceived barriers. BMJ Qual Saf 2018;27:928-36. 